\title{
Design and Simulation of a Reflectarray Antenna using New Cell for Different Beam Angles
}

\author{
Iman Aryanian $^{1}$, Abdolali Abdipour ${ }^{2}$, Gholamreza Moradi ${ }^{3}$ \\ 1, 2,3Department of Electrical Engineering, Amirkabir University of Technology, \\ Tehran, Iran \\ 1'iman_aryanian@aut.ac.ir, ${ }^{2}$ abdipour@aut.ac.ir, ${ }^{3}$ ghmoradi@aut.ac.ir
}

\begin{abstract}
In this paper a new cell is proposed for reflectarray antenna and is used to design the antenna to obtain to maximum gain and efficiency using phase synthesis in a frequency band of $11 \mathrm{GHz}$ up to $11.7 \mathrm{GHz}$ for different beam angles. The proposed cell is a double ring of hexagon which introduces multiple resonances which can provide more than 360 degrees phase variation by changing the loop size. Design method is based on phase-only algorithm where amplitude of the field on the reflectarray surface is forced by the feed. A $1.2 \mathrm{~m}$ reflectarray is designed for different beam directions. The results show maximum directivity of $42 d B$ and maximum efficiency of $73 \%$ for the required bandwidth. Focal length is $1.5 \mathrm{~m}$ which is set for maximum efficiency.
\end{abstract}

Keywords: loop element; phase-only algorithm; reflectarray antenna

\section{Introduction}

Printed reflectarray has some advantages compared to usual reflectors, three of which- that is, saving volume, simplifying the mechanical design, and applying easily to deployable reflectors are of great importance. A reflectarray antenna is a planar reflector made of microstrip patch arrays illuminated by a feed. The reflection phase from the reflectarray elements are designed so that the reflected energy from the array is collimated to form a main beam in a given direction or to generate a shaped beam in the coverage area. The phase of the reflected field can be changed by varying the size of the resonating patches $[1,2]$, by a transmission line of proper length connected [3,4] or aperture-coupled to the patches [5-7], or adding active elements to the antenna [8-11] like MEMs-based structures [12], varactor loaded patches [13, 14], PIN diodes [15-21] or liquid crystal based structures [22].

Square ring, circular ring, dual square rings, dual circular rings [23], multi cross loop [24], and combination of cross and rectangle loop elements [25] are example of ring elements used in previous works. In this paper a new unit cell is proposed and its phase response is obtained. The proposed cell is a double ring of hexagon which introduces multiple resonances with more than 360 degrees phase variation by changing the loop size. Proposed cell is used to design a high gain wideband reflectarray for different beam angles. To synthesis the radiation patterns using reflectarrays, a phase-only synthesis technique is used and the amplitude distribution is imposed by the feed.

\section{Reflectarray Design}

There are some aspects that must be taken into account in design of a reflectarray antenna, like the selection criteria for the phasing elements, the appropriate spacing to provide enough phase range and avoid the appearance of grating lobes and the geometrical definition of the antenna. First step to design a reflectarray is to select a good *Corresponding Author 
element. Different elements are proposed for reflectarray design. There are some parameters that should be considered to choose a proper element.

The element should be designed in a way that the slope of the reflected wave phase as a function of the elements' size becomes slower. This will result in a wider operational bandwidth and smaller susceptibility to manufacturing errors. Moreover, the reflected wave phase as a function of the elements' size should have more than 360 degrees range.

After selecting the proper element, the antenna can be designed in a four step process. First, the antenna size should be assessed by the desired gain. In the next step, location of the feed should be calculated to obtain maximum efficiency. Next, the field received on the antenna surface from the feed should be determined and lastly, the compensating phase of each element should be calculated to have a beam in a desired direction. After these four steps and choosing the length of each element, the antenna is designed and its gain can be calculated by $G=\eta \frac{4 \pi A}{\lambda^{2}}$, where $\mathrm{G}$ is the antenna gain, $\eta$ is the efficiency, A is the area of the antenna, and $\lambda$ is the wave length.

\subsection{Element Design}

The phase change versus element change should be calibrated which is done by full wave simulation in HFSS software. In this simulation the infinite array approach is used which gives a good approximation of the reflected phase from the element using Floquet modes. A new cell is used in this design which is a double ring of hexagon shown in Fig. 1. This cell element etched on a thick substrate; introduces multiple resonances that can provide more than 360 degrees phase variation by changing the loop size. This element is shown in Fig. 1, and its phase variation versus loop length change is shown in Fig. 2. The cell has the following parameters: cell size $=12 \mathrm{~mm}, \mathrm{w}=0.3 \mathrm{~mm}, \mathrm{~g}=0.4 \mathrm{~mm}$, and designed frequency is $\mathrm{f} 0=11.3 \mathrm{GHz}$. The proposed element is assumed to be supported by a substrate of thickness $3.175 \mathrm{~mm}$ and dielectric constant of 2.2 .

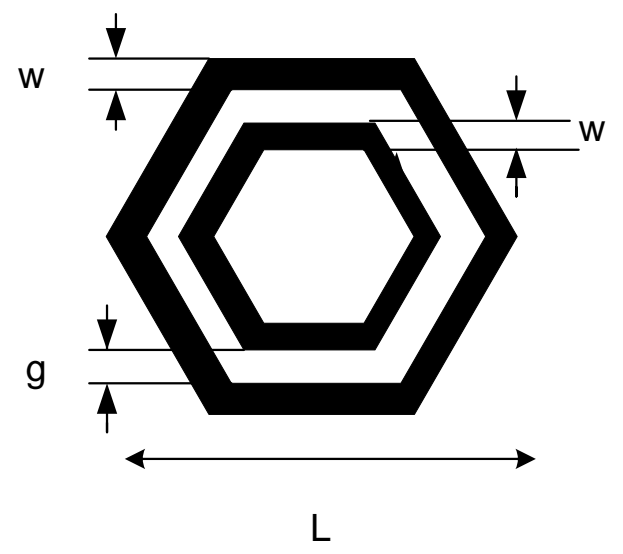

Figure 1. Cell Element 


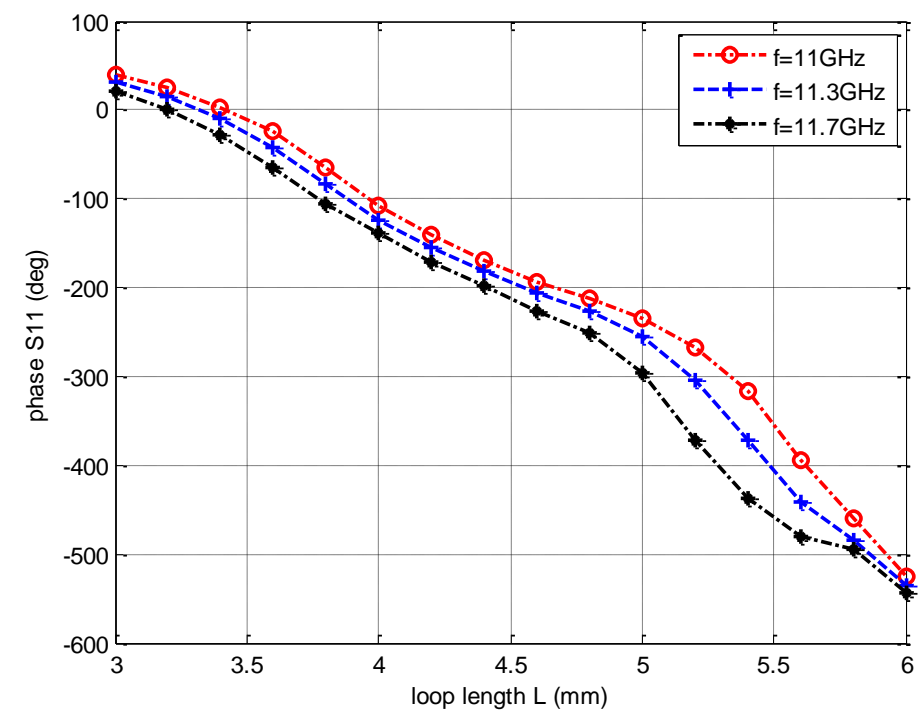

Figure 2. Phase of Simulated Reflected Wave against Element Loop Size at Different Frequencies in Frequency Band

\subsection{Calculating Size of the Antenna}

We select a rectangular antenna to increase the gain, and the size of the antenna is determined by [26]:

$G=\eta \frac{4 \pi A}{\lambda^{2}}$

For a gain of about $42 \mathrm{~dB}, \eta=.7$, and $\mathrm{f}=11.3 \mathrm{GHz}$, the required area of the antenna is $1.27 \mathrm{~m} 2$. The length of the antenna is determined from the square root of its area which is $1.13 \mathrm{~m}$. Also, number of the elements is calculated by dividing the antenna area to the basic element area. To diminish the grating lobes, the elements are linearly spaced with half a free space wavelength in both directions.

\subsection{Selecting Location of the Feed}

The focal length is set to reach to maximum efficiency. In other words, the efficiency is calculated for different feed locations and the best one is selected. The aperture efficiency can be defined as the product of the illumination and spillover efficiencies. The efficiency is calculated by integrating from the pattern. The illumination efficiency for a center fed reflectarray can be obtained in a closed form as [26]:

$\eta_{I}=\frac{\left[\left(\left(1+\cos ^{q+1} \theta_{e}\right) /(q+1)\right)+\left(\left(1-\cos ^{q} \theta_{e}\right) / q\right)\right]^{2}}{2 \tan ^{2} \theta_{e}\left[\left(1-\cos ^{2 q+1} \theta_{e}\right) /(2 q+1)\right]}$

and the spillover efficiency is given by [26]:

$\eta_{S}=1-\cos ^{2 q+1} \theta_{e}$

where $\mathrm{q}$ is the exponent of the feed pattern function represented by $\cos ^{q} \theta$, and $\theta_{e}$ is half of the subtend angle from the feed to the reflectarray aperture. (2) and (3) are calculated for a center fed circular antenna while we should integrate over a rectangular 
antenna. $\mathrm{q}$ is determined from the taper factor at the edges of the reflectarray, which is about $-10 \mathrm{~dB}$ for a focused beam ( Error! Reference source not found. ' Error! Reference source not found. , Error! Reference source not found.) and therefore, $q$ is 13.

Now, each element's position is computed and phase of the feed in these locations is determined by calculating the distance from the feed. Phase of the center element is considered zero and other elements phase is computed from the center element.

\subsection{Compensating the Phase}

Lastly, the Compensating phase of each element should be determine to have a focused beam in the desired direction. Now, consider our reflectarray contains $\mathrm{N}$ reflecting elements that are illuminated by a feed located at the focal point of the reflectarray. Considering a horn feed illuminating the individual patches of the reflectectarray, the excitation terms are proportional to the magnitude and phase of the electric field at the nth patch. It can be assumed that the amplitude of the reflected field is unchanged and only its phase is changing. So, one factor that affects the reflected field at each patch is the distance between the feed phase center and the n'th element phase center $\left(R_{n}\right)$. Furthermore, the feed has a certain angular taper over the reflectarray surface which can be included in the reflectarray pattern analysis by multiplying the relative complex excitation term by a raised cosine factor [26] that can be adjusted to match the pattern of the actual feed by choosing the proper q. The angular taper of the feed can be modeled as $\frac{e^{-j k R_{n}}}{R_{n}} \cos \theta_{n}^{q}$. Consequently, by multiplying the complex excitation term by the compensating phase factor $e^{-j k \psi_{n}}$ resulted from each element, the complex reflected field from each element can be expressed in the form:

$\frac{\cos \theta_{n}^{q}}{\left|R_{n}\right|} e^{-j\left(k R_{n}-\psi_{n}\right)}$

in which $\psi_{n}$ is the compensation phase of the n'th element.

The required phase shift at each element to produce a collimated beam in a given direction is [26]:

$\psi_{n}=k_{0}\left(R_{n}-\left(x_{n} \cos \phi_{b}+y_{n} \sin \phi_{b}\right) \sin \theta_{b}\right)$

where $\theta_{b}, \phi_{b}$ is the beam direction, $\mathrm{k} 0$ is the propagation constant in vacuum, and $\left(x_{n}, y_{n}\right)$ is the coordinates of element $\mathrm{n}$. Consequently, by considering the curve of phase change by element length change, length of each element can be determined from (5). To have a wideband antenna, the achieved phase in a frequency band should be optimized. Thus, we use a cost function to reduce the error in the frequency band which is:

$e(n)=\sum_{i=l, c, u}\left|\Phi^{\text {desired }}\left(f_{i}\right)(n)-\Phi^{\text {achieved }}\left(f_{i}\right)(n)\right|$

where $\Phi^{\text {desired }}\left(f_{i}\right)(m, n)$ is the desired phase in the location of element $\mathrm{n}$ obtained by (5) in the lower, centre, and upper frequency in the frequency band and $\Phi^{\text {achieved }}\left(f_{i}\right)(n)$ is the achieved phase from element $\mathrm{n}$ in the lower, centre, and upper frequency in the frequency band. This cost function compares the phase obtained from the element and desired phase and chooses the best element length which reduces the cost function. 


\section{Calculating the Gain}

After choosing the elements' length, antenna gain can be calculated. The antenna gain can be computed using the input power to the feed horn $P_{F}$ as [26]:

$G(\theta, \varphi)=\frac{4 \pi r^{2}}{2 \eta_{0} P_{F}}|E(\theta, \varphi)|^{2}$

where $\eta_{0}$ is the intrinsic impedance of the free space and $|E(\theta, \varphi)|$ is the amplitude of the field in far field and the input power of the feed horn is [26]:

$P_{F}=\frac{\pi}{\eta_{0} \lambda^{2}(2 q+1)}$

Moreover, electric field in the far field region is calculated as [26]:

$$
\begin{gathered}
\left|E_{X}\right|=\frac{k_{0}}{2 \pi}|S|\left|1+\frac{\left(\sqrt{1-u^{2}-v^{2}}-1\right) u^{2}}{u^{2}+v^{2}}\right| \\
\sum_{m, n} A_{x}^{m, n} \exp \left[j \phi_{x}^{m, n}+j k_{0}\left(u m d_{x}+v n d_{y}\right)\right]
\end{gathered}
$$

where $A_{x}^{m, n}$ and $\phi_{x}^{m, n}$ represent amplitude and phase of the reflected field on the $(\mathrm{m}, \mathrm{n})$ element and, $v=\sin \theta \sin \phi \quad, \quad u=\sin \theta \cos \phi \quad, \quad S=4 \sin (0.5 k u d) \sin (0.5 k v d) / k u v \quad$, and $d_{x}=d_{y}=12 \mathrm{~mm}$.

\section{Results}

We can see the pattern of the antenna designed for different beam direction in Fig. 3 up to Fig. 9. The antenna is designed for lower, center and upper frequency in the frequency band and the patterns are shown in frequencies of $11 \mathrm{GHz}, 11.3 \mathrm{GHz}$, and $11.7 \mathrm{GHz}$. Maximum gain of the antenna is $42 \mathrm{~dB}$ for angle of 0,10 and 20 degrees, and $41 \mathrm{~dB}$ for angle of 30 and 40 degrees, and $40 \mathrm{~dB}$ for angle of 50 degrees, and $38 \mathrm{~dB}$ for angle of 60 degrees. As the angle of broadside increases, the maximum gain decreases.

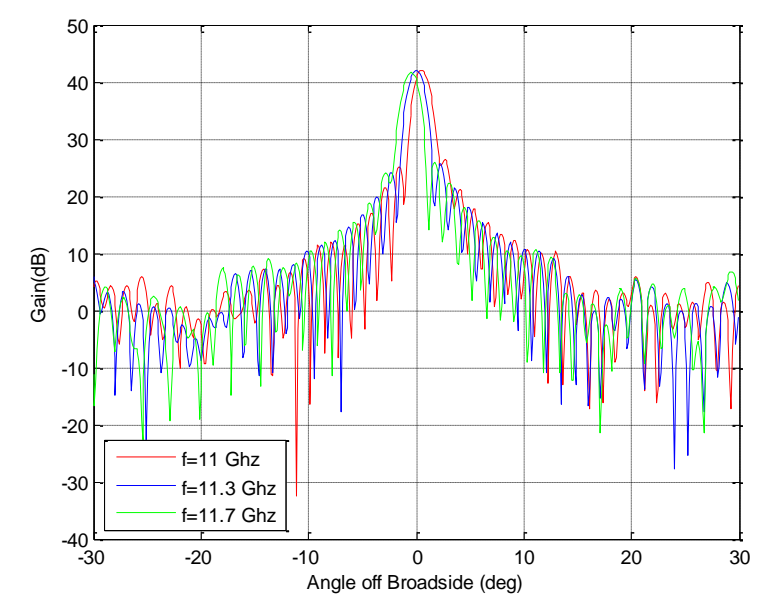

Figure 3. Radiation Pattern of the Antenna for Angle of Odeg 


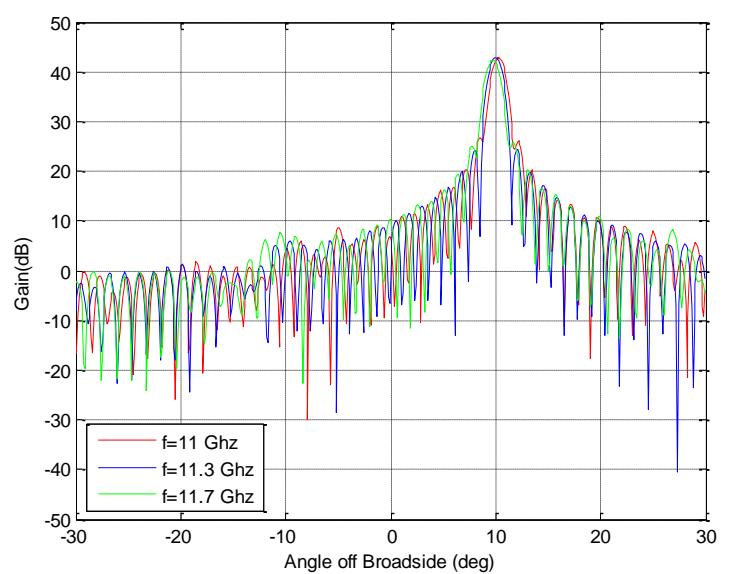

Figure 4. Radiation Pattern of the Antenna for Angle of $10 \mathrm{deg}$

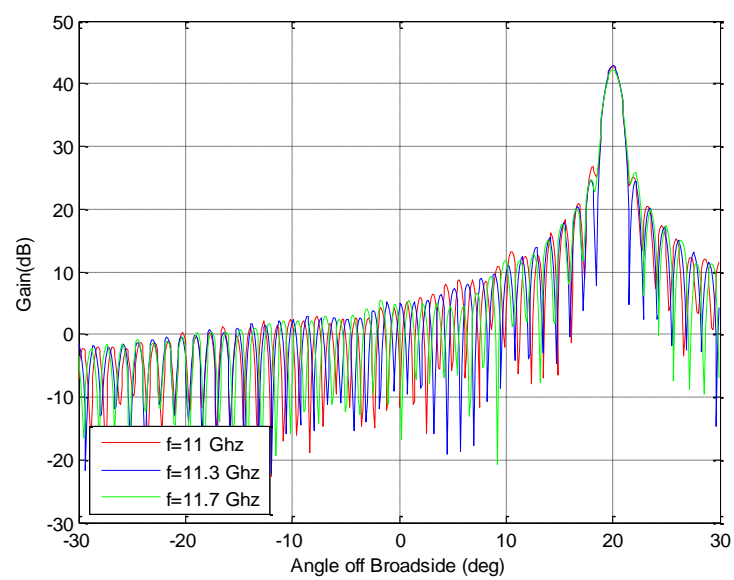

Figure 5. Radiation Pattern of the Antenna for Angle of $20 \mathrm{deg}$

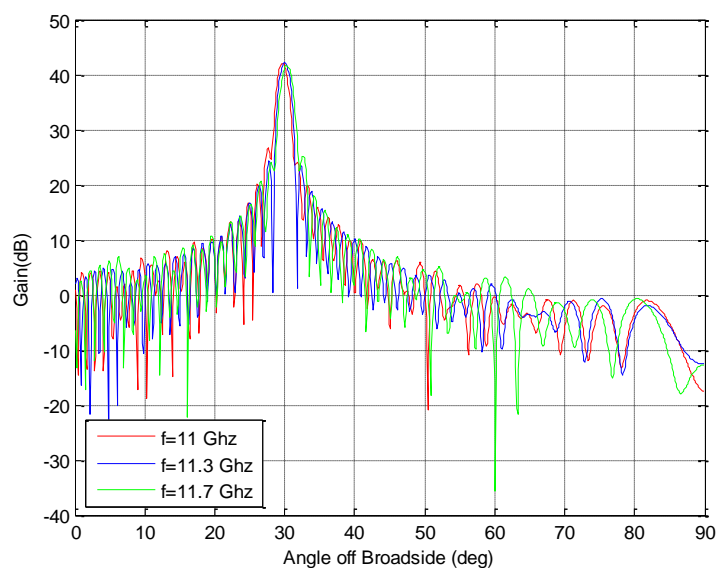

Figure 6. Radiation Pattern of the Antenna for Angle of $30 \mathrm{deg}$ 


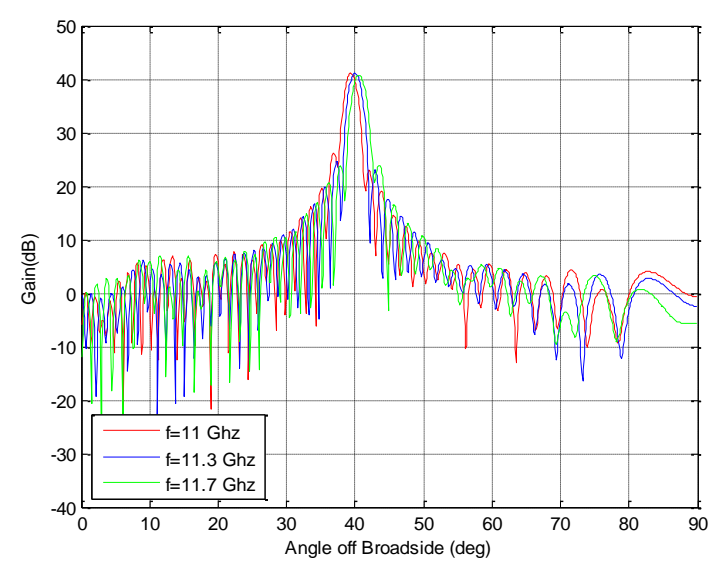

Figure 7. Radiation Pattern of the Antenna for Angle of $40 \mathrm{deg}$

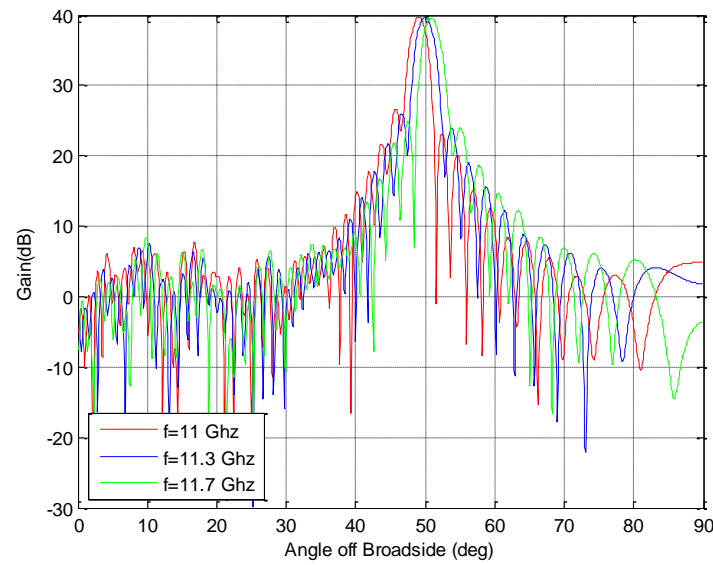

Figure 8. Radiation Pattern of the Antenna for Angle of $50 \mathrm{deg}$

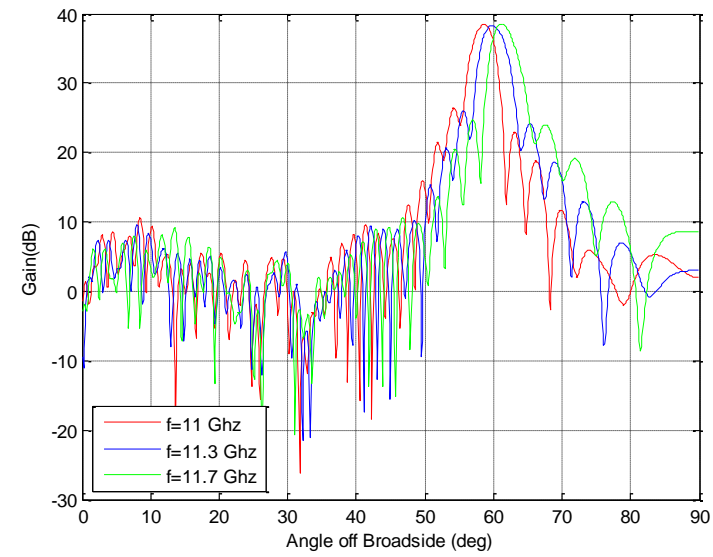

Figure 9. Radiation Pattern of the Antenna for Angle of $60 \mathrm{deg}$ 


\section{Conclusion}

A new loop element using dual hexagonal ring has been proposed to design reflectarray antenna which has a phase range of 566 degrees. Then, this element has been used to design a reflectarray antenna to have a high gain using phase only synthesis in a frequency band of $11 \mathrm{GHz}$ up to $11.7 \mathrm{GHz}$ where amplitude of the field on the reflectarray surface has been forced by the feed. Furthermore, a $1.2 \mathrm{~m}$ reflectarray has been designed for different beam directions with focal length of $1.5 \mathrm{~m}$ which is set for maximum efficiency of $73 \%$. The resulted radiation patterns show that the maximum directivity is $42 \mathrm{~dB}$ for the required bandwidth.

\section{Acknowledgments}

We thanks microwave/mm-wave and wireless communication research laboratory of Amirkabir University of Technology and Iran Telecommunication Research Center, ITRC.

\section{References}

[1] D. Pozar, and T. Metzler, "Analysis of a reflectarray antenna using microstrip patches of variable size," Electronics Letters, vol. 29, no. 8, (1993), pp. 657-658.

[2] P.-Y. Qin, Y. Guo, and A. Weily, "Broadband Reflectarray Antenna Using Sub-wavelength Elements Based on Double Square Meander-Line Rings,"(2016).

[3] H. Hasani, M. Kamyab, and A. Mirkamali, "Low cross-polarization reflectarray antenna," Antennas and Propagation, IEEE Transactions on, vol. 59, no. 5, (2011), pp. 1752-1756.

[4] R. S. Malfajani, and Z. Atlasbaf, "Design and implementation of a broadband single-layer reflectarray antenna with large-range linear phase elements," Antennas and Wireless Propagation Letters, IEEE, vol. 11, (2012), pp. 1442-1445.

[5] M. Bialkowski, and H. Song, "Dual linearly polarized reflectarray using aperture coupled microstrip patches." pp. 486-489.

[6] F. Venneri, S. Costanzo, G. Di Massa, and G. Amendola, "Aperture-coupled reflectarrays with enhanced bandwidth features," Journal of Electromagnetic Waves and Applications, vol. 22, no. 11-12, (2008), pp. 1527-1537.

[7] I. Aryanian, A. Abdipour, and G. Moradi, "Studying the nonlinear performance of an amplifying reflectarray antenna," International Journal of Microwave and Wireless Technologies, (2016), pp. 1-11.

[8] T. Makdissy, R. Gillard, E. Fourn, E. Girard, and H. Legay, "Phase-Shifting Cell for Dual Linearly Polarized Reflectarrays with Reconfigurable Potentialities,” (2013).

[9] F. Venneri, S. Costanzo, and G. Di Massa, "Design and validation of a reconfigurable single varactortuned reflectarray," Antennas and Propagation, IEEE Transactions on, vol. 61, no. 2, (2013), pp. 635645.

[10] I. Aryanian, A. Abdipour, and G. Moradi, "Nonlinear Analysis of Active Aperture Coupled Reflectarray Antenna Containing Varactor Diode," Applied Computational Electromagnetics Society Journal, vol. 30, no. 10, (2015).

[11] I. Aryanian, A. Abdipour, and G. Moradi, "Design fabrication and test of an X-band dual polarized aperture coupled reflectarray element for beam switching", (2016).

[12] O. Bayraktar, O. A. Civi, and T. Akin, "Beam switching reflectarray monolithically integrated with RF MEMS switches," Antennas and Propagation, IEEE Transactions on, vol. 60, no. 2, (2012), pp. 854862.

[13] M. Riel, and J. Laurin, "Design of an electronically beam scanning reflectarray using aperture-coupled elements," Antennas and Propagation, IEEE Transactions on, vol. 55, no. 5, (2007), pp. 1260-1266.

[14] F. Venneri, S. Costanzo, and G. Di Massa, "Reconfigurable aperture-coupled reflectarray element tuned by single varactor diode," Electronics Letters, vol. 48, no. 2, (2012), pp. 68-69.

[15] B. D. Nguyen, K. T. Pham, V.-S. Tran, L. Mai, N. Yonemoto, A. Kohmura, and S. Futatsumori, "Electronically tunable reflectarray element based on C-patch coupled to delay line," Electronics Letters, vol. 50, no. 16, (2014), pp. 1114-1116.

[16] S. Montori, F. Cacciamani, R. Vincenti Gatti, R. Sorrentino, G. Arista, C. Tienda Herrero, J. Encinar, and G. Toso, "A Transportable Reflectarray Antenna for Satellite Ku-band Emergency Communications."

[17] E. Carrasco, M. Barba, and J. A. Encinar, "X-band reflectarray antenna with switching-beam using pin diodes and gathered elements," Antennas and Propagation, IEEE Transactions on, vol. 60, no. 12, (2012), pp. 5700-5708 
[18] A. E. Martynyuk, J. Martinez Lopez, and N. A. Martynyuk, "Spiraphase-type reflectarrays based on loaded ring slot resonators," Antennas and Propagation, IEEE Transactions on, vol. 52, no. 1, (2004), pp. 142-153.

[19] H. Kamoda, T. Iwasaki, J. Tsumochi, T. Kuki, and O. Hashimoto, "60-GHz electronically reconfigurable large reflectarray using single-bit phase shifters," Antennas and Propagation, IEEE Transactions on, vol. 59, no. 7, (2011), pp. 2524-2531.

[20] E. Carrasco, J. A. Encinar, and M. Barba, "Dual linear polarized reflectarray element with true-time delay." pp. 3733-3737.

[21] R. Pereira, R. Gillard, R. Sauleau, P. Potier, T. Dousset, and X. Delestre, "Four-state dual polarisation unit-cells for reflectarray applications," Electronics letters, vol. 46, no. 11, (2010), pp. 742-743.

[22] G. Perez-Palomino, R. Florencio, J. A. Encinar, M. Barba, R. Dickie, R. Cahill, P. Baine, M. Bain, and R. R. Boix, "Accurate and Efficient Modeling to Calculate the Voltage Dependence of Liquid Crystal Based Reflectarray Cells," (2014).

[23] M. E. Bialkowski, and K. H. Sayidmarie, "Investigations into phase characteristics of a single-layer reflectarray employing patch or ring elements of variable size," Antennas and Propagation, IEEE Transactions on, vol. 56, no. 11, (2008), pp. 3366-3372.

[24] M. Chaharmir, and J. Shaker, "Broadband reflectarray with combination of cross and rectangle loop elements," Electronics Letters, vol. 44, no. 11, (2008), pp. 658-659.

[25] M. Chaharmir, J. Shaker, and H. Legay, "Broadband design of a single layer large reflectarray using multi cross loop elements," Antennas and Propagation, IEEE Transactions on, vol. 57, no. 10, (2009), pp. 3363-3366.

[26] J. Huang, Reflectarray antenna: Wiley Online Library, (2008). 
International Journal of Grid and Distributed Computing Vol. 9, No. 6 (2016) 